\title{
Comprehensive analysis of circRNA expression profiles and circRNA-associated competing endogenous RNA networks in IgA nephropathy
}

\author{
Haiyang Liu ${ }^{1}$, Di Liu ${ }^{1}$, Yexin Liu ${ }^{1}$, Ming Xia ${ }^{1}, \operatorname{Yan}_{\mathbf{L i}}{ }^{1}, \mathbf{M e i}_{\mathbf{L i}}{ }^{1}$, Hong Liu ${ }^{\text {Corresp. } 1}$ \\ ${ }^{1}$ Department of Nephrology, The Second Xiangya Hospital of Central University, Hunan Key Laboratory of Kidney Disease and Blood Purification, \\ Changsha, Hunan, China \\ Corresponding Author: Hong Liu \\ Email address: liuhong618@csu.edu.cn
}

Background. Immunoglobulin A nephropathy (IgAN) is immune-mediated primary glomerulonephritis, which is the most common reason leading to renal failure worldwide, the exact pathogenesis of IgAN is not well defined. Accumulating evidence indicates that circular RNAs (circRNAs) play crucial roles in the immune disease by involving in the competing endogenous RNA (ceRNA) network mechanism. At present, the studies of the circRNA profiles and circRNA-associated ceRNA networks in the IgAN are still scarce. This study aimed to elucidate the potential roles of circRNA-associated ceRNA networks of peripheral blood mononuclear cells (PBMCs) in IgAN patients. Method. CircRNA sequencing was used to identify the differential expressed circRNAs (DEcircRNAs) of PBMCs in IgAN and healthy controls; limma packages from data sets GSE25590 and GSE73953 in the Gene Expression Omnibus (GEO) database, were used to identify differentially expressed micro RNAs (miRNAs) and message RNAs (mRNAs). A circRNA-miRNA-mRNA ceRNA network was constructed to further investigate the mechanisms of IgAN. Then, $G O$ analysis and KEGG enrichment analyses were used to annotate the genes involved in the circRNAassociated ceRNA network. Further, Protein-protein interaction (PPI) networks were established to screen potential hub genes, by using Search Tool for the Retrieval of Interacting Genes/Proteins (STRING). Last, a quantitative real-time polymerase chain reaction (qRT-PCR) was applied to verify the hub genes in the ceRNA network.Result. A total of 145 circRNAs, 22 miRNAs, and 1117 mRNAs were differentially expressed in IgAN compared with controls $(P<0.05)$. A ceRNA network was constructed which contained 16 DEcircRNAs, 72 differential expressed mRNAs (DEmRNAs) and 11 differential expressed miRNAs (DEmiRNAs). KEGG pathway enrichment analysis illustrated the underlying biological functions of the ceRNA-associated genes, such as Nitrogen compound metabolic process, COPII-coated ER to Golgi transport vesicle, CAMP response element protein binding process $(P<0.01)$, meanwhile, Hepatitis $B, G n R H$ signaling, and Prion disease were 
the most significant enrichment $\mathrm{GO}$ terms $(\mathrm{P}<0.01)$. PPI network based on STRING analysis identified 4 potentially hub genes. Finally, Ankyrin Repeat And SOCS Box Containing 16 (ASB16), SEC24 homolog C, COPII coat complex component (SEC24C) were confirmed by qRT-PCR $(P<0.05)$ and were identified as the hub genes of the ceRNA network in our study.Conclusion. Our study identified a novel circRNA-mediated ceRNA regulatory network mechanisms in the pathogenesis of IgAN. 


\section{Comprehensive analysis of circRNA expression profiles and}

2 circRNA-associated competing endogenous RNA networks in IgA

3 nephropathy

4 Haiyang Liu ${ }^{1}$, Di Liu ${ }^{1}$, Yexin Liu ${ }^{1}$, Ming $\mathrm{Xia}^{1}$, Yan $\mathrm{Li}^{1}, \mathrm{Mei} \mathrm{Li}^{1}$, Hong Liu .

5

$6 \quad{ }^{1}$ Department of Nephrology, The Second Xiangya Hospital of Central University, Hunan Key

7 Laboratory of Kidney Disease and Blood Purification, Changsha, Hunan, China

8

9 Corresponding Author:

10 Hong Liu ${ }^{1}$

11 No. 139 Renmin Middle Rd, Changsha, Hunan, 410011, China

12 Email address: liuhong618@,csu.edu.cn

13

14 


\section{Comprehensive analysis of circRNA expression profiles and 37 circRNA-associated competing endogenous RNA networks in IgA nephropathy}

39

40

41

42

43

44

45

46

47

48

49

50

51

52

53

54

55

56

57

58

59

60

61

62

63

64

65

66

67

68

69

70

71

72

73

74

75

76

77

78

\section{Abstract}

Background. Immunoglobulin A nephropathy (IgAN) is immune-mediated primary glomerulonephritis, which is the most common reason leading to renal failure worldwide, the exact pathogenesis of IgAN is not well defined. Accumulating evidence indicates that circular RNAs (circRNAs) play crucial roles in the immune disease by involving in the competing endogenous RNA (ceRNA) network mechanism. At present, the studies of the circRNA profiles and circRNA-associated ceRNA networks in the IgAN are still scarce. This study aimed to elucidate the potential roles of circRNA-associated ceRNA networks of peripheral blood mononuclear cells (PBMCs) in IgAN patients.

Method. CircRNA sequencing was used to identify the differential expressed circRNAs (DEcircRNAs) of PBMCs in IgAN and healthy controls; limma packages from data sets GSE25590 and GSE73953 in the Gene Expression Omnibus (GEO) database, were used to identify differentially expressed micro RNAs (miRNAs) and message RNAs (mRNAs). A circRNA-miRNA-mRNA ceRNA network was constructed to further investigate the mechanisms of IgAN. Then, GO analysis and KEGG enrichment analyses were used to annotate the genes involved in the circRNA-associated ceRNA network. Further, Protein-protein interaction (PPI) networks were established to screen potential hub genes, by using Search Tool for the Retrieval of Interacting Genes/Proteins (STRING). Last, a quantitative real-time polymerase chain reaction (qRT-PCR) was applied to verify the hub genes in the ceRNA network.

Result. A total of 145 circRNAs, 22 miRNAs, and 1117 mRNAs were differentially expressed in IgAN compared with controls $(\mathrm{P}<0.05)$. A ceRNA network was constructed which contained 16 DEcircRNAs, 72 differential expressed mRNAs (DEmRNAs) and 11 differential expressed miRNAs (DEmiRNAs). KEGG pathway enrichment analysis illustrated the underlying biological functions of the ceRNA-associated genes, such as Nitrogen compound metabolic process, COPII-coated ER to Golgi transport vesicle, CAMP response element protein binding process $(\mathrm{P}<0.01)$, meanwhile, Hepatitis $\mathrm{B}, \mathrm{GnRH}$ signaling, and Prion disease were the most significant enrichment GO terms $(\mathrm{P}<0.01)$. PPI network based on STRING analysis identified 4 potentially hub genes. Finally, Ankyrin Repeat And SOCS Box Containing 16 (ASB16), SEC24 homolog C, COPII coat complex component (SEC24C) were confirmed by qRT-PCR $(\mathrm{P}<0.05)$ and were identified as the hub genes of the ceRNA network in our study.

Conclusion. Our study identified a novel circRNA-mediated ceRNA regulatory network mechanisms in the pathogenesis of IgAN.

\section{Introduction}

IgA nephropathy (IgAN) has a global incidence exceeding 1.5 per 100000 persons, which is the most common type of glomerulonephritis worldwide (Wyatt \& Julian. 2013). The clinical manifestation often presents as macroscopic hematuria following an upper respiratory or gastrointestinal infections. Diagnosed of IgAN is exclusively identified by renal biopsy with 
characteristics of predominant IgA deposition in the glomerular mesangium (Wyatt \& Julian. 2013), and the 10-year risk of end-stage renal disease varies widely from $5 \%$ to $60 \%$ (Reich et al., 2007). In the exploring of pathogenesis in IgAN, many mechanisms including the four-hit hypothesis had been proposed in the academic: the unknown upstream inducing the production of galactose-deficient IgA1(Gd-IgA1), which were identified as auto-antigen and targeted by autoantibody, formatted as immune complex in the circulation, deposited in kidney thus resulting in following renal injuries (Suzuki et al., 2011). Noticeably, recurrence of IgAN is 50\% over 5 years after kidney transplantation (Mulay et al., 2009), while it's reported that several IgAN cases experienced a recovery after bone marrow transplantation (Park et al., 2008), suggesting IgAN appears to be a systemic immune disease in which the kidneys are damaged as innocent bystanders (Wyatt \& Julian. 2013).

Recently, circular RNAs (circRNAs) arouse attention as one of the factors correlated with immune response, they are special types of single-stranded non-coding RNA which has a closed feature and without 3'poly (A) and 5'-cap structure (Chen. 2020; Yan., 2020). CircRNA is formed by the back-splicing of pre-mRNAs processing, in which a downstream $5^{\prime}$ splice site is joined to an upstream $3^{\prime}$ splice site in reverse order across an exon or exon. Investigator recently found that after B cell infected with Kaposi's sarcoma herpesvirus, hundreds of differentially expressed human circRNAs were identified, which suggests those newly expressed circRNAs may work as an antiviral mechanism by suppressing crucial viral genes (Tagawa et al., 2018). The expression of circRNAs is usually stable, for it was resistant to exonucleases, and largely exported to the cytoplasm (Chen. 2020). The harboring miRNA recognition elements (MREs) of circRNAs could competitively bind to certain miRNAs, and regulate miRNA-mediated downstream target gene silencing at the post-transcriptional level, thus participate in the manipulation process of the target genes and been described involved in the competing endogenous RNA (ceRNA) hypothesis (Thomson \& Dinger. 2016 ).

The ceRNA hypothesis has been demonstrated involved in kidney disease, for example, novel_circ-0004153/rno-miR-1443p/Gpnmb ceRNA relationship has been reported involved in acute kidney injury of rat model (Cheng et al., 2019); circHLA-C could function as a sponge to decoying miRNA-150, then promoted renal fibrosis by regulating fibrosis-associated genes in lupus nephritis (Luan et al., 2018). However, the studies of the circRNA profiles and circRNAassociated ceRNA networks in the IgAN are still scarce, therefore, exploring the expression of non-coding RNAs including circRNAs and miRNAs may bring potential opportunities in the understanding mechanism of IgAN.

PBMCs are blood cells with round nuclei that encompass a heterogeneous cell population (with $70-90 \%$ T cells, B cells, and NK cells)( Sallustio et al., 2019), which originate from hematopoietic stem cells that reside in the bone marrow. In this study, PBMCs from IgAN patients and healthy controls were collected and sequenced with next-generation technology, and combined analysis with the genes expression profiles in the GEO database, a circRNA associated ceRNA network of IgAN was established (Fig. 1), aiming to comprehensively investigate the potential circRNA related molecular mechanism in IgAN.

\section{Materials \& Methods}

\section{PBMCs collection and RNA preparation}

PeerJ reviewing PDF | (2020:06:50257:1:1:NEW 15 Oct 2020) 
122 This research was approved by the Ethics Committee of the Second Xiangya Hospital (IRB2018-

123 S095). Participants were from The Second Xiangya Hospital, and all of them had provided

124 signed informed consent. The diagnosis of IgAN was based on renal biopsy with the deposition

125 of IgA in the glomerular mesangium (Wyatt \& Julian. 2013), patients with any other systemic

126 diseases or secondary IgAN were excluded. Peripheral blood from 3 IgAN patients and 3 healthy

127 controls were centrifuged at $2000 \mathrm{rpm}$ for $10 \mathrm{~min}$, PBS was added to diluted the remaining

128 blood, by Ficoll-Hypaque (GE Healthcare) gradient (2000 rpm for $30 \mathrm{~min}$ ) to isolate the PBMCs

129 by density separation. The isolated PBMCs were used for RNA isolation. Then, total RNA was

130 extracted using Trizol reagent (Invitrogen, CA, USA) following the manufacturer's procedure.

131 The total RNA quantity and purity were analyzed using Bioanalyzer 2100 and RNA 6000 Nano

132 LabChip Kit (Agilent, CA, USA) with RNA integrity number $>7.0$. Approximately 5 ug of total

133 RNA were used to deplete ribosomal RNA according to the Ribo-Zero ${ }^{\text {TM }}$ rRNA Removal Kit

134 (Illumina, San Diego, USA). After removing ribosomal RNAs, the remaining RNAs were

135 fragmented into small pieces using divalent cations under high temperatures. Then the cleaved

136 RNA fragments were reverse-transcribed to create the cDNA, which was next used to

137 synthesized U-labeled second-stranded DNAs with E. coli DNA polymerase I, RNase H and

138 dUTP. An A-base is then added to the blunt ends of each strand, preparing them for ligation to

139 the indexed adapters. Each adapter contains a T-base overhang for ligating the adapter to the A-

140 tailed fragmented DNA. Single-or dual-index adapters are ligated to the fragments, and size

141 selection was performed with AMPureXP beads. After the heat-labile UDG enzyme treatment of

142 the U-labeled second-stranded DNAs, the ligated products were amplified with PCR with the

143 following conditions: initial denaturation at $95^{\circ} \mathrm{C}$ for $3 \mathrm{~min} ; 8$ cycles of denaturation at $98^{\circ} \mathrm{C}$ for

$14415 \mathrm{~s}$, annealing at $60^{\circ} \mathrm{C}$ for $15 \mathrm{~s}$, and extension at $72^{\circ} \mathrm{C}$ for $30 \mathrm{~s}$; and then final extension at $72^{\circ} \mathrm{C}$ for

$1455 \mathrm{~min}$. The average insert size for the final cDNA library was $300 \mathrm{bp} \mathrm{(} \pm 50 \mathrm{bp}$ ).

\section{CircRNA sequencing and microarray data download}

147 First, we performed the paired-end sequencing (PE150) for the library on Illumina Hiseq 4000 (LC

148 Science Co., LTD., Hangzhou, China) following the vendor's recommended protocol. Cutadapt

149 (Martin et al., 2011) was used to remove the reads that contained adaptor contamination, low

150 quality bases and undetermined bases. Then sequence quality was verified using FastQC

151 (http://www.bioinformatics.babraham.ac.uk/projects/fastqc/). We used Bowtie2 (Langmead et al.,

152 2012) and Hisat2 (Kim et al., 2015) to map reads to the genome of Homo sapiens (GRCh38).

153 Remaining reads (unmapped reads) were still mapped to genome using tophat-fusion (Kim et al.,

154 2011). CIRCExplorer2 (Zhang et al., 2014 and Zhang et al., 2016) was used to de novo assemble

155 the mapped reads to circRNAs at first. Then, back splicing reads were identified in unmapped

156 reads by tophat-fusion. All samples generated unique circular RNAs ( step1: the two ends of splice

157 sites must be GU/AG; step 2: mismatch $\leq 2$; step 3: back spliced junctions read $\geq 1$; step 4: the

158 distance between two splice sites on the genome is not more than $100 \mathrm{~kb}$ ).

159 We next performed an electronic search in GEO (http:/www.ncbi.nlm.nih.gov/geo/) database 160 using the keywords "IgA nephropathy, IgA nephritis, or Berger's disease" to identify studies 161 involving samples with PBMCs from IgAN patients. Studies were included if they met the 162 following criteria: (1) patients were diagnosed with biopsy-proven IgAN; (2) case-control studies 163 and the number of cases and controls in each dataset must be $\geq 2$; (3) all datasets were genome- 
164

165

166

167

168

169

170

171

172

173

174

175

176

177

178

179

180

181

182

183

184

185

186

187

188

189

190

191

192

193

194

195

196

197

198

199

200

201

202

203

204

205

wide; (4) complete microarray raw data were available. We excluded any animal or duplicated studies. The whole-genome raw expression data of included studies were downloaded from the GEO dataset. Therefore, the GSE73953 (mRNA) and GSE25590 (miRNA) microarray datasets that contain IgAN's PBMC related information were selected. GSE73953 including 15 IgAN patients, 2 healthy controls (Nagasawa et al., 2016), which was based on the GPL4133 platform (Agilent-014850 Whole Human Genome Microarray 4x44K G4112F). GSE25590 contained 7 IgAN patients and 7 matched healthy samples (Serino et al., 2012) and was based on the GPL7731 platform (Agilent-019118 Human miRNA Microarray 2.0 G4470B).

\section{Differential expression analysis of circRNAs, miRNAs, and mRNAs}

The unit of measurement for circRNA is Fragment Per Kilobase of exon per Million fragments mapped (FPKM). The differentially expressed circRNAs were selected with $\log 2$ (fold change) $>1$ or $\log _{2}$ (fold change) $<-1\left(\left|\log _{2}(\mathrm{FC})\right|>1\right)$ and with statistical significance $(\mathrm{P}-\mathrm{value}<0.05)$ by R package-edgeR (Robinson et al., 2010).

The expression status of GSE73953 and GSE25590 were obtained by R-package agimicrorna processing, normalizeBetweenArrays in the Limma package was used to normalize the expression values in the quartile (Ritchie et al., 2015). Finally, $\log 2$ transformation was used to obtain standardized expression values. Significance of differential miRNAs and mRNAs expression were defined by $\left|\log _{2}(\mathrm{FC})\right|>1$, q-value $<0.05$ and $\mathrm{P}$-value $<0.05$. The heat maps were made by Pheatmap software, and the volcano plot was made by Ggplot 2 software.

\section{Function enrichment analysis of the differential expression genes.}

To investigate the potential function of the differential expression genes (DEGs) in the PBMCs of IgAN, GO (http://www.geneontology.org/) and KEGG (http://www.kegg.jp/) pathway enrichment analysis of the identified DEGs were performed. The identified DEGs were classified in terms of the biological process (BP), molecular function (MF), and cellular component (CC) categories (Ashburner et al., 2000). KEGG analysis was utilized to interpret the potential functions and pathways of the DEGs (Kanehisa et al., 2010).

\section{CeRNA network construction and functional analysis of the genes in the network}

We analyzed the circRNAs and mRNAs which are expressed in significantly different levels between the IgAN patients and the control group. The sequences of circRNAs, miRNAs, and mRNAs were screened to search the potential MREs. We used 4 databases including miRanda (http://www.microrna.org/microrna/home.do), PITA (http://genie.weizmann.ac.il/pubs/mir07/mir07_dyn_data.html), RNA22 (http://cm.jefferson.edu/rna22/Interactive/) and TarPmiR (http://hulab.ucf.edu/research/projects/miRNA/TarPmiR/) to find miRNAs-circRNAs relationship, only overlapping genes were selected as candidate. Next, we used 5 kinds of databases including MiRDB (http://mirdb.org/), TargetScan (http://www.targetscan.org/), miRanda, miRMap (https://mirmap.ezlab.org/) and miTarBase (http://mirtarbase.mbc.nctu.edu.tw/) to screen mRNAs corresponding targets of miRNAs, and retained the interaction genes which are identified at least 4 of the databases. The circRNAmiRNA-mRNA regulatory network was constructed using a combination of circRNA-miRNA and miRNA-mRNA interaction. Finally, the network was visualized and mapped using Cytoscape v3.7.0 software. All of the DEGs coming from co-expression and prediction ceRNA

PeerJ reviewing PDF | (2020:06:50257:1:1:NEW 15 Oct 2020) 
206

207

208

209

210

211

212

213

214

215

216

217

218

219

220

221

222

223

224

225

226

227

228

229

230

231

232

233

234

235

236

237

238

239

240

241

242

243

244

245

network were united for genes functional annotation and enrichment analysis, to investigate the potential molecular mechanism of the genes in IgAN.

\section{Protein-protein Interaction network analysis and qRT-PCR of the hub genes.}

To assess the interactions between DEGs in the ceRNA network, we used the Search Tool for the Retrieval of Interacting Genes (STRING, https://stringdb.org/) online tool, which can provide comprehensive interactions among proteins and genes, and established a Protein-Protein Interaction (PPI) network of our ceRNA network. A Required Confidence (combined score) $>0.7$ was used to visualize the established PPI network. Then we used Cytoscape to analyze the topology of genes in the PPI network, the central proteins in the network are found by using the scale-free nature of the interaction PPI network. Also, $4 \mathrm{IgAN}$ patients and 4 healthy controls in the Second Xiangya Hospitals that signed informed consent, were recruited for qRT-PCR validation of the expression of hub genes. The way of PBMCs collection and RNA extraction were mentioned previously, the qRT-PCR reaction was performed using the UltraSYBR Mixture (Cwbiotech, China), in the Pikoreal PCR Detection System (Thermo, USA) with the following conditions: $95^{\circ} \mathrm{C}$ for 10 mins, then followed by 40 cycles of $95^{\circ} \mathrm{C}$ for $15 \mathrm{~s}$ and $60^{\circ} \mathrm{C}$ for the $30 \mathrm{~s}$. The quantitative primers of ankyrin repeat and SOCS box containing 16 (ASB16), major histocompatibility complex, class I, B (HLA-B), tripartite motif containing 21(TRIM21), SEC24 homolog C, COPII coat complex component (SEC24C), were designed and synthesized by Sangon Biotech (Sangon Biotech, China) and are listed in Table S1, $\beta$-actin was used as the house-keeping gene for normalization, qRT-PCR relative fold change results were calculated using the $2-\Delta \Delta \mathrm{Ct}$ method.

\section{Results}

\section{Overview of circRNA-Seq and identification of DEcircRNAs}

After the removal of low-quality reads, adapters, poly-N $>5 \%$, and other contaminant-containing reads from the raw data, clean reads of circRNA-sequencing (circRNA-seq) were obtained.

In the present study, a total of 16,940 circRNA transcripts were identified, among them, 145 circRNAs were differentially expressed in the IgAN patients compared to the healthy controls, including 112 up-regulated and 33 down-regulated circRNAs. The basic characteristic of the top 10 DEcircRNAs are listed in Table 1, the most up-regulated circRNA was hsa_circ_0038725 with $6.03 \log _{2}(\mathrm{FC})$, and the most down-regulated circRNA was circRNA11137 (gene symbol: EMB) with -6.01 $\log _{2}(\mathrm{FC})$, those DEcircRNAs were used for the subsequent analysis (Table $S 2$ ). A heat-map of DEcircRNAs was illustrated in Fig. $2 \mathrm{~A}$.

\section{Identification of DEmiRNAs and DEmRNAs}

A total of 22 DEmiRNAs were screened from the GSE25590 dataset, including 18 up-regulated and 4 down-regulated miRNAs in IgAN patients (Table S3). In the GSE73953 dataset, a total of 1117 DEmRNAs were identified, including 522 up-regulated and 595 down-regulated mRNAs in IgAN patients (Table S4). The DEmiRNAs and DEmRNAs between IgAN patients and controls are illustrated as heat-map in Fig. $2 B C$. The statistical analysis of the differential expressed circRNAs, miRNAs and mRNAs were summarized in Table 2. A volcano plot was utilized to 
246 visualize the statistical significance of DEGs between the IgAN patients and the controls (Fig.

$2473 A)$.

\section{Pathway enrichment analysis of the DEGs}

249 KEGG analysis illustrated those down-regulated DEGs were mainly enrichment in the NOD-like

250 receptor signaling pathway (hsa04621, 19 genes were enriched with $\mathrm{P}<10^{-6}$ ), Fc gamma

251 Receptor-mediated phagocytosis (hsa04666, 13 genes were enriched with $\mathrm{P}<10^{-6}$ ), Measles

252 pathway (hsa05162, 15 genes were enriched with $\mathrm{P}<10^{-5}$ ), etc (Fig. 3B). Those upregulated

253 DEGs were mainly enriched in the Mineral absorption pathway (hsa04978, 8 genes were

254

255

256

257

258

259 enriched with $\mathrm{P}<10^{-5}$ ), Maturity onset diabetes of the young pathway (hsa04950, 4 genes were enriched with $\mathrm{P}<10^{-4}$ ), Linoleic acid metabolism pathway (hsa00591, 4 genes were enriched with $\mathrm{P}<10^{-4}$ ), etc (Fig. 3C). The results of GO analysis shows that the down-regulated DEGs were enriched in functions associated with Leukocyte activation involved in immune response (GO:0002366, 55 genes were enriched with $\mathrm{P}<10^{-15}$ ), Cytoplasm (GO:0005737, 382 genes were enriched with $\mathrm{P}<10^{-16}$ ), Identical protein binding (GO:0042802, 80 genes were enriched with

260

261

262

263 $\left.\mathrm{P}<10^{-5}\right)$. The upregulated DEGs were enriched in function associated with Cellular response to copper ion (GO:0071280, 10 genes were enriched with $\mathrm{P}<10^{-11}$ ), Integral component of postsynaptic specialization membrane (GO:0099060, 8 genes were enriched with $\mathrm{P}<10^{-5}$ ), Hormone activity (GO:0005179, 8 genes were enriched with $\mathrm{P}<10^{-4}$ ) (Table S5 and Fig. S1).

Construction of the ceRNA network and functional annotation of genes in ceRNA network

266

267

268

269

270

271

272

273

274

275

276

277

278

279

280

281

282

283

284

285

286

287

According to the ceRNA hypothesis, the members of ceRNA (circRNAs, miRNAs, and mRNAs) compete for the same miRNA response elements (MREs) to regulate each other. To establish a circRNA-miRNA-mRNA ceRNA network, we found 32 DEmiRNAs-DEcircRNAs pairs according to the circRNAs-miRNAs corresponding relationship, which overlapped in 4 databases including miRanda, PITA, RNA22, and TarPmiR. (Fig. 4A)

Then, we used 5 kinds of databases including MiRDB, TargetScan, miRanda, miRMap and miTarBase to identify mRNAs corresponding targets of miRNAs, and 12 DEmRNAsDEmiRNAs relationship pairs which are overlapping at least 4 of the databases. Overlapping datasets were visualized using Venn diagrams (Fig. 4B).

Finally, we constructed a ceRNA network based on 16 circRNA nodes, 11 miRNA nodes, and 72 mRNA nodes (Fig. 4C). These RNA interactions may serve as a novel perspective for exploring the underlying mechanism of IgAN. More details are listed in Tables S6.

All of the related genes coming from the established ceRNA network were united for genes functional annotation enrichment analysis to investigate the potential roles of these differentially expressed circRNAs and mRNAs (Table S7). The KEGG analysis showed that the pathway of genes in the network were mostly related with Hepatitis B (hsa05161, 4 genes were enriched with $\mathrm{P}=0.003$ ), GnRH signaling pathway (hsa04912, 3 genes were enriched with $\mathrm{P}=0.005$ ), and Prion disease (hsa05020, 2 genes were enriched with $\mathrm{P}=0.007$ ) (Fig. 4D). The most significant biological process is the regulation of Nitrogen compound metabolic process (GO:0051171, 35 genes were enriched with $\mathrm{P}<10^{-4}$ ), Vesicle targeting (GO:0006903, 4 genes were enriched with $\mathrm{P}<10^{-4}$ ), Vesicle budding from the membrane (GO:0006900, 4 genes were enriched with $\mathrm{P}<10^{-4}$ ) (Fig. 4E). The most relevant cellular component are COPII-coated ER to Golgi transport vesicle 
288

289

290

291

292

293

294

295

296

297

298

299

300

301

302

303

304

305

306

307

308

309

310

311

312

313

314

315

316

317

318

319

320

321

322

323

324

325

326

327

328

329

(GO:0030134, 5 genes were enriched with $\left.\mathrm{P}<10^{-4}\right)$, Coated vesicle (GO:0030135, 7 genes were enriched with $\mathrm{P}<10^{-4}$ ), and Heterochromatin (GO:0000792, 4 genes were enriched with $\mathrm{P}<10^{-3}$ ) (Fig. 4F). CAMP response element binding (GO:0008140, 2 genes were enriched with $\mathrm{P}<10^{-3}$ ), Non-membrane spanning protein tyrosine kinase activity (GO:0004715, 3 genes were enriched with $\mathrm{P}<10^{-5}$ ), and Calcium-dependent phospholipid binding (GO:0005544, 3 genes were enriched with $\mathrm{P}=0.001$ ) were the most enrichment molecular function (Fig .4G).

\section{Protein-protein Interaction network analysis and validation of the hub genes}

Furthermore, the genes were selected which has potential functional associated (in the STRING database) with other genes in the ceRNA network, and then established PPI networks. The PPI network involving 7 nodes and 4 edges (Fig. 5A). Among then, ASB16, HLA-B, TRIM21, SEC24C were relatively highly connected in the PPI network (associated with more than 3 molecules) and were considered to be the potential hub genes (He \& Zhang. 2006). qRT-PCR was utilized to validates those hub genes in the PPI networks (Fig. $5 B / C / D / E)$, as a result, ASB16 was confirmed significant up-regulated $(\mathrm{P}=0.015)$ (Fig. 5B) and SEC24C was significantly downregulated in IgAN patients $(\mathrm{P}=0.021)$ (Fig. 5D). This indicates the up-regulated ASB16 and downregulated SEC24C may play important roles in the pathogenesis and pathological process in IgAN.

\section{Discussion}

In the presenting study, we successfully identified DEcircRNAs in PBMCs of $\operatorname{IgAN}(\mathrm{P}<0.05)$, combined with the identified DEmiRNAs and DEmRNAs $(\mathrm{P}<0.05)$ in the RNA profiles in the GEO database, a ceRNA network was constructed with 16 DEcircRNAs, 11 DEmiRNAs, and 72 DEmRNAs according to the ceRNA hypothesis. The function analysis revealed the genes in the ceRNA network mainly involved in the Nitrogen compound metabolic process, COPII-coated ER to Golgi transport vesicle, CAMP response element protein binding process in the GO term annotation, meanwhile, it's showed that the Hepatitis B, GnRH signaling pathway, Prion disease and Human T-cell leukemia virus 1 infection were the most enriched KEGG pathway, and interestingly, 3 of them were closely related to the virus infection. The protein interaction analysis revealed that ASB16, SEC24C were considered as hub genes in the ceRNA networks. Therefore, our results have firstly revealed the potential mechanism of circRNAs-associated ceRNA networks in IgAN.

Since the 1990s, many studies have found that competing endogenous circRNAs could work as endogenous miRNA 'sponges', which take part in regulating miRNA related downstream gene expression (Chen. 2020). Ongoing investigation illustrated that significant expression of circRNAs was observed in platelets, hematopoietic progenitor cell differentiation into lymphoid and myeloid cells, and been observed they have functionally involved in neuronal function, cell proliferation, and innate immunity. But the pathological function of circRNA is still largely unknown, for instances, rheumatoid arthritis is a chronic and systemic autoimmune disease with unknown etiology (Coutant and Miossec. 2020), multiple groups have independently identified several differentially expressed circRNAs in PBMCs between rheumatoid arthritis patients and healthy controls (Wen JT et al., 2020; Yang X et al., 2019). Similarly, systemic lupus erythematosus (SLE) in one of the autoimmune disease and could also be manifested as glomerulonephritis, several circRNAs including hsa_circ_0044235 have been found to be

PeerJ reviewing PDF | (2020:06:50257:1:1:NEW 15 Oct 2020) 
330 significantly decreased in PBMCs, and are proposed as potential negative biomarkers for SLE 331 diagnosis (Luo Q et al., 2019).

332 In the field of IgAN, several studies have investigated the non-coding RNA in PBMCs. For 333 examples, UDP-N-acetyl- $\alpha$-d-galactosamine: polypeptide $\mathrm{N}$-acetylgalactosaminyltransferase 2

334 (GALNT2) could catalysis the attachment of N-acetylgalactosamine (GalNAc) to the

335 serine/threonine of the hinge region of the molecule, which functions in the early step in O336 linked glycosylation in IgA1, the significantly down-regulated GALNT2 in the PBMCs thus correlated with the Gd-IgA1 level in IgAN, Serino et al had reported that miRNA let-7b not only involving influence the expression of GALNT2, it could also play roles as a biomarker for detecting primary IgAN (Serino et al., 2016). In the presenting study, we have first time identified 112 up-regulated and 33 down-regulated circRNAs in IgAN, the interaction regulatory mechanism of the related circRNA have been carefully predicted. Consistent with the literature, the GALNT2 were significantly down-regulated in the PBMCs of IgAN in our study, while the hsa_circ_0070562、hsa_circ_0066719、hsa_circ_0073237 and circRNA3302 were identified as ceRNAs of miR765, therefore could participate in the manipulation the expression of target GALNT2. However, unexpectedly all the 3 circRNAs were up-regulated in the study, therefore didn't follow the classic circRNA (down in IgAN)-miRNA (up in IgAN)-mRNA (down in IgAN) interaction mechanism, therefore, it remains unknown whether the regulation of GLANT2 in IgAN is influenced by the circRNAs in PBMCs. It's should be mentioned that Thomson \& Dinge used to discuss in 2016 (Thomson \& Dinger. 2016 ), the underlying argument opinion which against the ceRNA hypothesis is that, the change in expression of an individual noncoding RNA may only impact relatively a small fraction of the target mRNA abundance. Therefore, the real mechanism of ceRNA interaction needs further molecular biology experiments, to carefully verify this non-coding factors on the molecules in the disease, such as the previous literature, using knock-out or overexpression method to illustrated a TGF- $\beta / \mathrm{Smad} 3$ interacting-lncRNA inhibits renal fibrogenesis in IgAN (Wang et al., 2018).

Next, we identified the hub genes of the ceRNA network. Normally most proteins interact with only a few other proteins, while a small number of proteins that have many interaction partners (the genes encoding them, namely hub genes) (He \& Zhang. 2006), and usually play important roles in the protein network, exploring of the hub genes may bring further insights into the presented ceRNA network. In our study, ASB16 and SEC24C were confirmed by qRT-PCR to have the same gene expression direction as detected by microarray, thus were identified as potentially the hub genes of the ceRNA networks. ASB16 is a member of the ankyrin repeat and SOCS box-containing (ASB) family of proteins, which may be a substrate-recognition component of E3 ubiquitin-protein ligase complex that mediates the ubiquitination and subsequent proteasomal degradation of target proteins (Liu P et al., 2019); SEC24C is a subunit of COPII complex, which is the coat protein complex responsible for vesicle budding from the endoplasmic reticulum (ER), and plays a role in shaping the vesicle (Subramanian et al., 2019), as well as in cargo selection and concentration, It's been reported SEC24C were significantly upregulated during $\mathrm{B}$ cell differentiation into a plasma cell and was assumed associated with the antibody-producing related preprocessing (Kirk et al., 2010).

371 In our study, the functional analysis of the DEGs in the PBMCs according to the genes profiles

372 GSE73953 had shown, the NOD-like receptor signaling pathway, Fc gamma R-mediated

373 phagocytosis, Mineral_absorption pathway were the most enriched pathway. In another study 
374 that had comprehensively analyzed 3 microarray data of PBMC in IgAN (Liu D et al., 2019), the

375 KEGG enrichment pathway analysis was confirmed with enriched in human T-cell leukemia

376 virus 1 infection, but also including proteoglycans in cancer and intestinal immune network for

377 IgA production. However, noticeably, our study had suggested that the genes in the circRNA-

378 associated ceRNA network were relatively more enriched in virus infection-related pathway. The

379 KEGG analysis indicated that the Hepatitis B, Prion disease, T-cell leukemia virus 1 infection,

380 and cytomegalovirus infection were significantly enriched in the genes of the ceRNA network.

381

382

383 It's well known that viruses could manipulate host gene expression, and among the various mechanism of this pathological process, the most efficient way is a non-coding RNAs strategy (Tycowski et al., 2015). Many kinds of research have revealed that viruses produce non-coding RNAs could serve as miRNA sponges, play as an adaptation to the ceRNA, and increased

385

386

387

388

389

390

391

392

393

394

395

396

397

398

399

400

401

402

403

404

405

406

407

408

409

410

411

412

413

414

415

416

417

418

419 ceRNA activity (Tycowski et al., 2015). But the mechanism investigation of virus-related ceRNA in IgAN was less explored up till now. In the related literature, the relationship between IgAN and HBV has been often noticed, it's reported 17-18\% of overall IgAN patients in China were HBV carriers (Zhao., 2020). Presently it's unknown that, whether the humoral immune response inducing by HBAg-HBAb immune complex causing the dysregulation of IgA producing or the mesangial cell injuries directly causing by HBV in situ were most involved in the pathogenesis of IgAN. While in another way, considering the prevalence of HBV infection could reach 5-7.99\% of the population in China, therefore, whether IgAN was secondary to HBV infection, or presenting as a geographical coincidence, both of them should be further carefully demonstrated. Besides, previous research of IgAN has revealed that in vitro EBVtransformed peripheral-blood cells from healthy individuals produce almost exclusively IgA1 subclass. In a recent study of Zachova et al (Zachova et al., 2020), which indicates EBV infection may be involved in the pathogenesis of IgAN. They found that B cells and their IgA ${ }^{+}$ subpopulation in peripheral blood of IgAN patients displayed a significantly higher frequency of EBV infection compared to the controls and displayed increased expression of homing receptors for targeting the upper respiratory tract. Similarly, in the animal model of IgAN, by oral immunization with the Sendai virus, a parainfluenza virus likewise to human respiratory tract viruses can induce IgAN in mice (Suzuki \& Suzuki. 2018). These in vivo and in vitro phenotypes had a raised speculation, that different types of viruses infection have shared some similar molecular biological processes, which aroused response of circRNAs expression and involved in the disease progression of IgAN.

Moreover, the GO enrichment analysis has shown that the circRNAs-associated genes' most related cellular component were intracellular membrane-bounded organelle and cytoplasm, especially the process associated with vesicle transport between the ER to Golgi. Although the molecule function of intracellular vesicle transport is relatively less discuss in IgAN, however, it's naturally closely related to the immunoglobulin's processing (Kirk et al., 2010). For instance, the C1GALT1, which function as a crucial enzyme of process IgA1 galactosylation, normally C1GALT1 is synthesized in ER under the help of molecular chaperone Cosmc, and it's necessary to be packaged in vesicles and transport to Golgi then finish the galactosylation of IgA1 in Golgi (Cummings. 2019). In IgAN, the significant deficiency of C1GALT1 (expression or/and activity) have resulted in the elevated of Gd-IgA1, and directly correlated with serum levels of Gd-IgA1 in IgAN patients which have been demonstrated by many works of literature (Lai et al., 2016). In our previous study, Wang et al (Wang et al., 2019) have illustrated the Golgi matrix protein 130 plays the role of docking C1GALT1 vesicles in Golgi, the dysregulation with this molecular process had been demonstrated associated with Gd-IgA1 in IgAN, and firstly

Peer) reviewing PDF | (2020:06:50257:1:1:NEW 15 Oct 2020) 
420 aroused the attention about the molecular process of ER-Golgi transportation in IgAN.

421 Combined with our findings according to the ceRNA network, exploring the virus infection-

422 related intracellular vesicle transport mechanism might be an interesting direction of IgAN in the

423 future.

424 Lastly, our study was designed as combined with the patient's data from Italian, Japanese and

425 Chinese populations, for better avoiding the bias brought by single-center sample, but in another

426 way, the different race' sample sources bring the heterogeneity challenges to the comprehensive

427 analysis of genes profile and validation of hub genes. For example, the molecular in this ceRNA

428 network was relatively less than other ceRNA comprehensively studies, and we couldn't find

429

430

431

432

433

434

435

ceRNA interaction pair with certain order (up-down-up or down-up-down) to validates the

potential associated relationship corresponding with GALNT, ASB16 or SEC24C. The small sample size of the hub gene verification is also the one of limitations of this study, validation of a larger sample size will be needed, and exploring the potential capability of circRNAs as

biomarkers of IgAN would be a research in the future. Future research should further identify the hub molecules with therapeutic potential in IgAN and conducted the overexpression or inhibition experiments of related ceRNAs, and observing the reciprocal relationship of target genes, for a

436 better understanding of the molecular pathogenesis of IgAN.

437

438

439

Conclusions

440

In summary, we successfully identified IgAN associated circRNAs using RNA-seq analysis, and elucidated the circRNA-associated ceRNA networks of IgAN through the integrated analysis of RNA expression profile. To our knowledge, this is the first report examining the expression of circRNAs in IgAN. These findings had expanded our understanding of circRNAs-associated

442

443 ceRNA networks in IgAN, a future exploring the related molecular regulatory mechanism will be

444 needed for a better understanding IgAN.

445

446

Acknowledgements

447

We would like to thank Wei Cheng (The Second Xiangya Hospital, Central South University)

448

for her kindly advice on the manuscript, her insights on ceRNA network mechanism have greatly

449 improved this work.

450

451

References

452

Ashburner M, Ball CA, Blake JA, Botstein D, Butler H, Cherry JM, Davis AP, Dolinski K,

453 Dwight SS, Eppig JT, Harris MA, Hill DP, Issel-Tarver L, Kasarskis A, Lewis S, Matese JC,

454

455

Richardson JE, Ringwald M, Rubin GM, and Sherlock G. 2000. Gene ontology: tool for the unification of biology. The Gene Ontology Consortium. Nat Genet 25:25-29.

456 DOI:10.1038/75556

457 Chen LL. 2020. The expanding regulatory mechanisms and cellular functions of circular RNAs.

458 Nat Rev Mol Cell Biol. Online ahead of print. Available at https://doi.org/10.1038/s41580-020-

459 0243-y (accessed 21 June 2020)

Peer) reviewing PDF | (2020:06:50257:1:1:NEW 15 Oct 2020) 
460 Cheng W, Li XW, Xiao YQ, Duan SB. 2019. Non-coding RNA-Associated ceRNA Networks 461 in a New Contrast-Induced Acute Kidney Injury Rat Model. Mol Ther Nucleic Acids 17:102-

462 112. DOI:10.1016/j.omtn.2019.05.011

463 Coutant F, Miossec P. 2020. Evolving concepts of the pathogenesis of rheumatoid arthritis with 464 focus on the early and late stages. Curr Opin Rheumatol 32(1):57-63.

465 DOI:10.1097/BOR.0000000000000664

466 Cummings RD. 2019. "Stuck on sugars - how carbohydrates regulate cell adhesion, recognition, 467 and signaling". Glycoconj J 36:241-257. DOI:10.1007/s10719-019-09876-0

468 He X, and Zhang J. 2006. Why do hubs tend to be essential in protein networks? PLoS Genet 469 2:e88. DOI:10.1371/journal.pgen.0020088

470 Kanehisa M, Goto S, Furumichi M, Tanabe M, and Hirakawa M. 2010. KEGG for representation 471 and analysis of molecular networks involving diseases and drugs. Nucleic Acids Res 38:D355472 360. DOI:10.1093/nar/gkp896

473 Kim D, Langmead B, Salzberg SL. 2015. HISAT: a fast spliced aligner with low memory 474 requirements. Nat Methods 12(4):357-360. DOI:10.1038/nmeth.3317

475 Kim D, Salzberg SL. 2011. TopHat-Fusion: an algorithm for discovery of novel fusion 476 transcripts. Genome Biol 12(8):R72. DOI:10.1186/gb-2011-12-8-r72

477 Kirk SJ, Cliff JM, Thomas JA, and Ward TH. 2010. Biogenesis of secretory organelles during B 478 cell differentiation. J Leukoc Biol 87:245-255. DOI:10.1189/jlb.1208774

479 Lai KN, Tang SC, Schena FP, Novak J, Tomino Y, Fogo AB, and Glassock RJ. 2016. IgA 480 nephropathy. Nat Rev Dis Primers 2:16001. DOI:10.1038/nrdp.2016.1

481 Langmead B, Salzberg SL. 2012. Fast gapped-read alignment with Bowtie 2. Nat Methods 482 9(4):357-359. DOI:10.1038/nmeth.1923

483 Liu D, Xia M, Liu Y, Tan X, He L, Liu Y, Chen G, and Liu H. 2020. The upregulation of miR$48498-5 p$ affects the glycosylation of IgA1 through cytokines in IgA nephropathy. Int

485 Immunopharmacol 82:106362. DOI:10.1016/j.intimp.2020.106362

486 Liu P, Verhaar AP, and Peppelenbosch MP. 2019. Signaling Size: Ankyrin and SOCS Box487 Containing ASB E3 Ligases in Action. Trends Biochem Sci 44:64-74.

488 DOI:10.1016/j.tibs.2018.10.003

489 Liu Y, Liu X, Jia J, Zheng J, Yan T. 2019. Comprehensive analysis of aberrantly expressed 490 profiles of mRNA and its relationship with serum galactose-deficient IgA1 level in IgA 491 nephropathy 17(1):320. DOI: 10.1186/s12967-019-2064-3.

492 Luan J, Jiao C, Kong W, Fu J, Qu W, Chen Y, Zhu X, Zeng Y, Guo G, Qi H, Yao L, Pi J, Wang 493 L, and Zhou H. 2018. circHLA-C Plays an Important Role in Lupus Nephritis by Sponging miR494 150. Mol Ther Nucleic Acids 10:245-253. DOI:10.1016/j.omtn.2017.12.006

495 Luo Q, Zhang L, Li X, Fu B and Li J. 2019. Identification of circular rnas hsa_circ_0044235 496 and hsa_circ_0068367 as novel biomarkers for systemic lupus erythematosus. International 497 Journal of Molecular Medicine 44(4), 1462-1472. DOI: 10.3892/ijmm.2019.4302 
498 Martin M. 2011. Cutadapt removes adapter sequences from high-throughput sequencing reads.

499 Embenet Journal 17:1. DOI: 10.14806/ej.17.1.200

500 Mulay AV, van Walraven C, and Knoll GA. 2009. Impact of immunosuppressive medication on 501 the risk of renal allograft failure due to recurrent glomerulonephritis. Am J Transplant 9:804-

502 811. DOI:10.1111/j.1600-6143.2009.02554.x

503 Nagasawa Y, Okuzaki D, Muso E, Yamamoto R, Shinzawa M, Iwasaki Y, Iwatani H, Nakanishi 504 T, Isaka Y, and Nojima H. 2016. IFI27 Is a Useful Genetic Marker for Diagnosis of

505 Immunoglobulin A Nephropathy and Membranous Nephropathy Using Peripheral Blood. PLoS

506 One 11:e0153252. DOI:10.1371/journal.pone.0153252

507 Park EK, Jeon JS, Noh HJ, Won JH, and Park HS. 2008. Complete remission of IgA

508 nephropathy after bone marrow transplantation for acute myeloid leukaemia. NDT Plus 1:420-

509 422. DOI:10.1093/ndtplus/sfn147

510 Reich HN, Troyanov S, Scholey JW, Cattran DC, and Toronto Glomerulonephritis R. 2007.

511 Remission of proteinuria improves prognosis in IgA nephropathy. J Am Soc Nephrol 18:3177-

512 3183. DOI:10.1681/ASN.2007050526

513 Ritchie ME, Phipson B, Wu D, Hu Y, Law CW, Shi W, and Smyth GK. 2015. limma powers

514 differential expression analyses for RNA-sequencing and microarray studies. Nucleic Acids Res

515 43:e47. DOI:10.1093/nar/gkv007

516 Robinson MD, McCarthy DJ, Smyth GK. 2010. edgeR: a Bioconductor package for differential

517 expression analysis of digital gene expression data. Bioinformatics 26(1):139-140.

518 DOI:10.1093/bioinformatics/btp616

519 Sallustio F, Curci C, Di Leo V, Gallone A, Pesce F, and Gesualdo L. 2019. A New Vision of IgA

520 Nephropathy: The Missing Link. Int J Mol Sci 21(1):189. DOI:10.3390/ijms21010189

521 Selvaskandan H, Pawluczyk I, and Barratt J. 2018. MicroRNAs: a new avenue to understand, 522 investigate and treat immunoglobulin A nephropathy? Clin Kidney J 11:29-37.

523 DOI:10.1093/ckj/sfx096

524 Serino G, Pesce F, Sallustio F, De Palma G, Cox SN, Curci C, Zaza G, Lai KN, Leung JC, Tang 525 SC, Papagianni A, Stangou M, Goumenos D, Gerolymos M, Takahashi K, Yuzawa Y, 526 Maruyama S, Imai E, and Schena FP. 2016. In a retrospective international study, circulating $527 \mathrm{miR}-148 \mathrm{~b}$ and let-7b were found to be serum markers for detecting primary IgA nephropathy.

528 Kidney Int 89:683-692. DOI:10.1038/ki.2015.333

529 Serino G, Sallustio F, Cox SN, Pesce F, and Schena FP. 2012. Abnormal miR-148b expression 530 promotes aberrant glycosylation of IgA1 in IgA nephropathy. J Am Soc Nephrol 23:814-824. 531 DOI:10.1681/ASN.2011060567

532 Subramanian A, Capalbo A, Iyengar NR, Rizzo R, di Campli A, Di Martino R, Lo Monte M, 533 Beccari AR, Yerudkar A, Del Vecchio C, Glielmo L, Turacchio G, Pirozzi M, Kim SG, Henklein 534 P, Cancino J, Parashuraman S, Diviani D, Fanelli F, Sallese M, and Luini A. 2019. Auto535 regulation of Secretory Flux by Sensing and Responding to the Folded Cargo Protein Load in the 536 Endoplasmic Reticulum. Cell 176:1461-1476 e1423. DOI:10.1016/j.cell.2019.01.035 
537 Suzuki H, and Suzuki Y. 2018. Murine Models of Human IgA Nephropathy. Semin Nephrol

538 38:513-520. DOI:10.1016/j.semnephrol.2018.05.021

539 Suzuki H, Kiryluk K, Novak J, Moldoveanu Z, Herr AB, Renfrow MB, Wyatt RJ, Scolari F,

540 Mestecky J, Gharavi AG and Julian BA. 2011. The pathophysiology of IgA nephropathy. J Am

541 Soc Nephrol 22:1795-803. DOI:10.1681/ASN.2011050464

542 Tagawa T, Gao S, Koparde VN, Gonzalez M, Spouge JL, Serquina AP, Lurain K, Ramaswami

543 R, Uldrick TS, Yarchoan R, and Ziegelbauer JM. 2018. Discovery of Kaposi's sarcoma

544 herpesvirus-encoded circular RNAs and a human antiviral circular RNA. Proc Natl Acad Sci U S

545 A 115:12805-12810. DOI:10.1073/pnas.1816183115

546 Thomson DW and Dinger ME. 2016. Endogenous microRNA sponges: evidence and

547 controversy. Nat Rev Genet 17:272-283. DOI:10.1038/nrg.2016.20

548 Tycowski KT, Guo YE, Lee N, Moss WN, Vallery TK, Xie M and Steitz JA. 2015. Viral

549 noncoding RNAs: more surprises. Genes Dev 29(6):567-84. DOI: 10.1101/gad.259077.115

550 Wang C, Ye M, Zhao Q, Xia M, Liu D, He L, Chen G, Peng Y, and Liu H. 2019. Loss of the 551 Golgi Matrix Protein 130 Cause Aberrant IgA1 Glycosylation in IgA Nephropathy. Am J

552 Nephrol 49:307-316. DOI:10.1159/000499110

553 Wang P, Luo ML, Song E, Zhou Z, Ma T, Wang J, Jia N, Wang G, Nie S, Liu Y, and Hou F. 554 2018. Long noncoding RNA lnc-TSI inhibits renal fibrogenesis by negatively regulating the

555 TGF-beta/Smad3 pathway. Sci Transl Med 10. DOI:10.1126/scitranslmed.aat2039

556 Wen JT, Liu J, Zhang PH, Jiang H, Xin L, Wan L, Sun Y, Huang D, Sun YQ, Long Y, Zhang Y, 557 Bao BX and Sun GH. 2020. RNA-seq reveals the circular RNA and miRNA expression profile 558 of peripheral blood mononuclear cells in patients with rheumatoid arthritis. Bioscience reports 559 40(4), BSR20193160. DOI:10.1042/BSR20193160

560 Wyatt RJ, and Julian BA. 2013. IgA nephropathy. N Engl J Med 368:2402-2414.

561 DOI:10.1056/NEJMra1206793

562 Yan L, Chen YG. 2020. Circular RNAs in Immune Response and Viral Infection. Trends

563 Biochem Sci. Online ahead of print. Available at https://doi.org/10.1016/j.tibs.2020.08.006

564 (accessed 21 Sep 2020)

565 Yang X, Li JY, Wu YZ, Ni B and Zhang B. 2019. Aberrant dysregulated circular RNAs in the

566

567

568 peripheral blood mononuclear cells of patients with rheumatoid arthritis revealed by RNA sequencing: novel diagnostic markers for RA. Scand J Clin Lab Invest 79(8):551-559.

569 Zachova K, Kosztyu P, Zadrazil J, Matousovic K, Vondrak K, Hubacek P, Julian BA, 570 Moldoveanu Z, Novak Z, Kostovcikova K, Raska M, and Mestecky J. 2020. Role of Epstein-

571 Barr Virus in Pathogenesis and Racial Distribution of IgA Nephropathy. Front Immunol 11:267.

572 DOI:10.3389/fimmu.2020.00267

573 Zhang XO, Dong R, Zhang Y, Zhang JL, Luo Z, Zhang J, Chen LL, Yang L. 2016. Diverse 574 alternative back-splicing and alternative splicing landscape of circular RNAs. Genome Res

575 26(9):1277-1287. DOI:10.1101/gr.202895.115 
576 Zhang XO, Wang HB, Zhang Y, Lu X, Chen LL, Yang L. 2014. Complementary sequence-

577 mediated exon circularization. Cell 159(1):134-147. DOI:10.1016/j.cell.2014.09.001

578 Zhao L, Peng L, Yang D, Chen S, Lan Z, Zhu X, Yuan S, Chen G, Liu Y, Liu H. 2020.

579 Immunostaining of galactose-deficient IgA1 by KM55 is not specific for immunoglobulin A

580 nephropathy. Clin Immunol 217:108483. DOI: 10.1016/j.clim.2020.108483.

581

582 


\section{Figure 1 (on next page)}

Main steps of the construction of the circRNA-associated ceRNA network in IgAN.

Step 1: The circRNA expression profiles of PBMCs in IgAN patients and healthy controls were explored by high-throughput sequencing, expression profiles of miRNAs and mRNAs were obtained from the data sets in the GEO database. Step 2: Significantly differential circRNAs, miRNAs and mRNAs were identified, the GO and KEGG enrichment analysis were conducted to reveal the functions of differential expressed mRNAs. Step 3: The ceRNA interaction relationships were predicted using online tools, then constructed the ceRNA network. Steps 4: Functional analysis of the related genes in the ceRNA network. Identification hub genes of the ceRNA network and validate the expression with qRT-PCR. IgAN: IgA nephropathy. DEcircRNA: differentially expressed circularRNA. DEmiRNA: differentially expressed microRNA. DEmRNA: differentially expressed mRNA. PBMCs: peripheral blood mononuclear cells. CeRNA: competing endogenous RNA. GO: Gene Ontology. KEGG: Kyoto Encyclopedia of Genes and Genomes. 


\section{Figure 2}

Heatmaps of differential expressed circRNAs, miRNAs and mRNAs.

Cluster analysis of expression of (A)circRNAs, (B)miRNAs and (C)mRNAs. Red and blue: increased and decreased expression, respectively. Columns represent different samples, rows indicates different genes. Significantly differential circRNA were defined by $\left|\log _{2}(F C)\right|$ $>1$ and P-value $<0.05$, the differential expression miRNAs and mRNAs were defined by $\| \log _{2}$ $(\mathrm{FC}) \mid>1$, P-value $<0.05$ and $\mathrm{q}$ - value $<0.05$

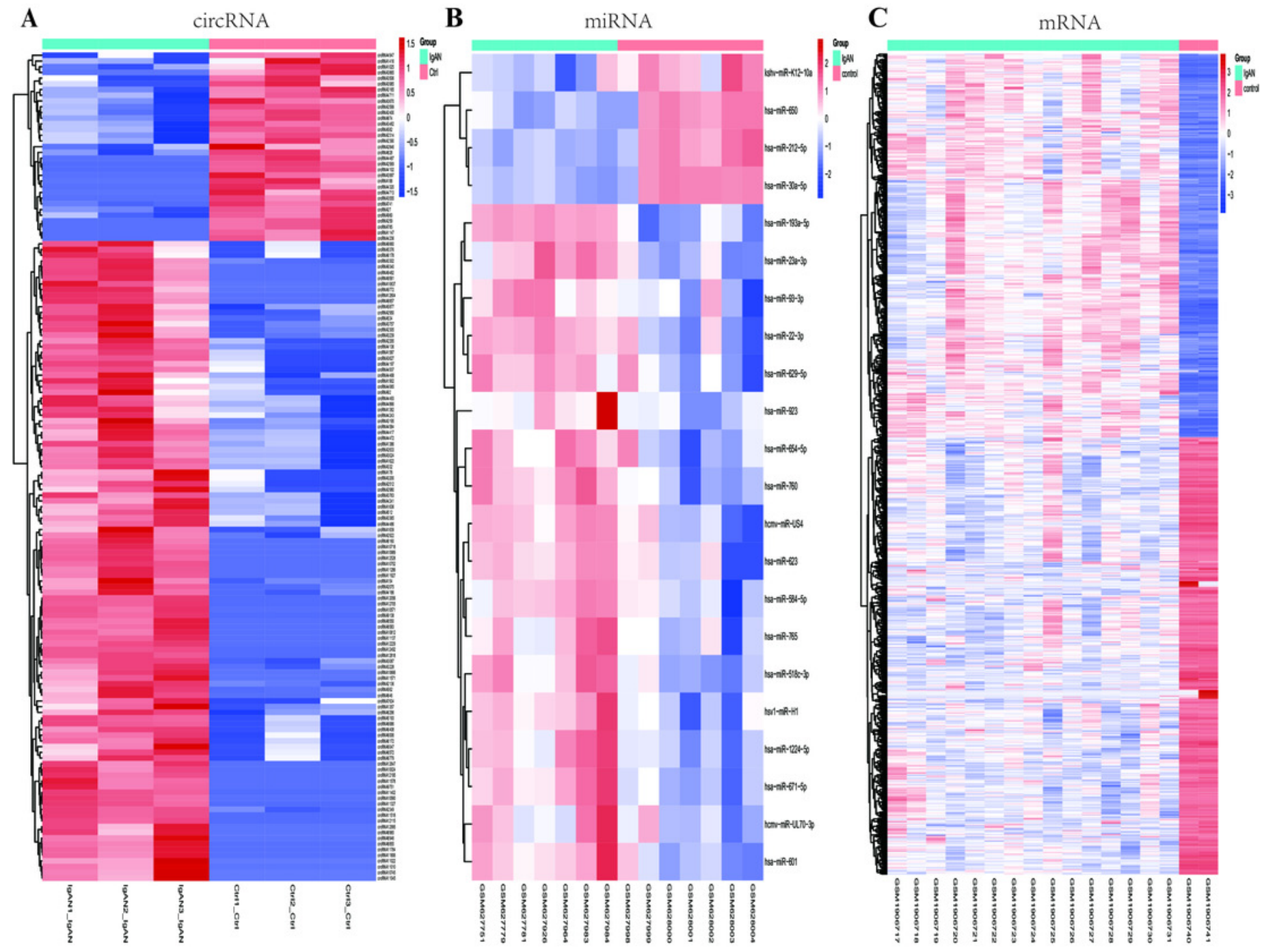




\section{Figure 3}

Volcano plot and top 10 enrichment KEGG pathways for DEGs.

(A) Volcano plot for the DEGs in data set GSE73953. The $x$-axis indicates the $\log _{2}(F C)$, and the $y$-axis indicates the log10 (q-value). The red dots represent up-regulated genes, and the blue dots represent down-regulated genes. The black dots represent the genes expressed without significant differences. (B) Top 10 enrichment KEGG pathways for down-regulated DEGs, and (C) up-regulate DEGs, the $x$-axis shows the names of the pathway names, the $y$-axis shows enrichment score. DEGs: differentially expressed genes.

A

IgAN vs control

- up (522) - middle (18632) - down (595)

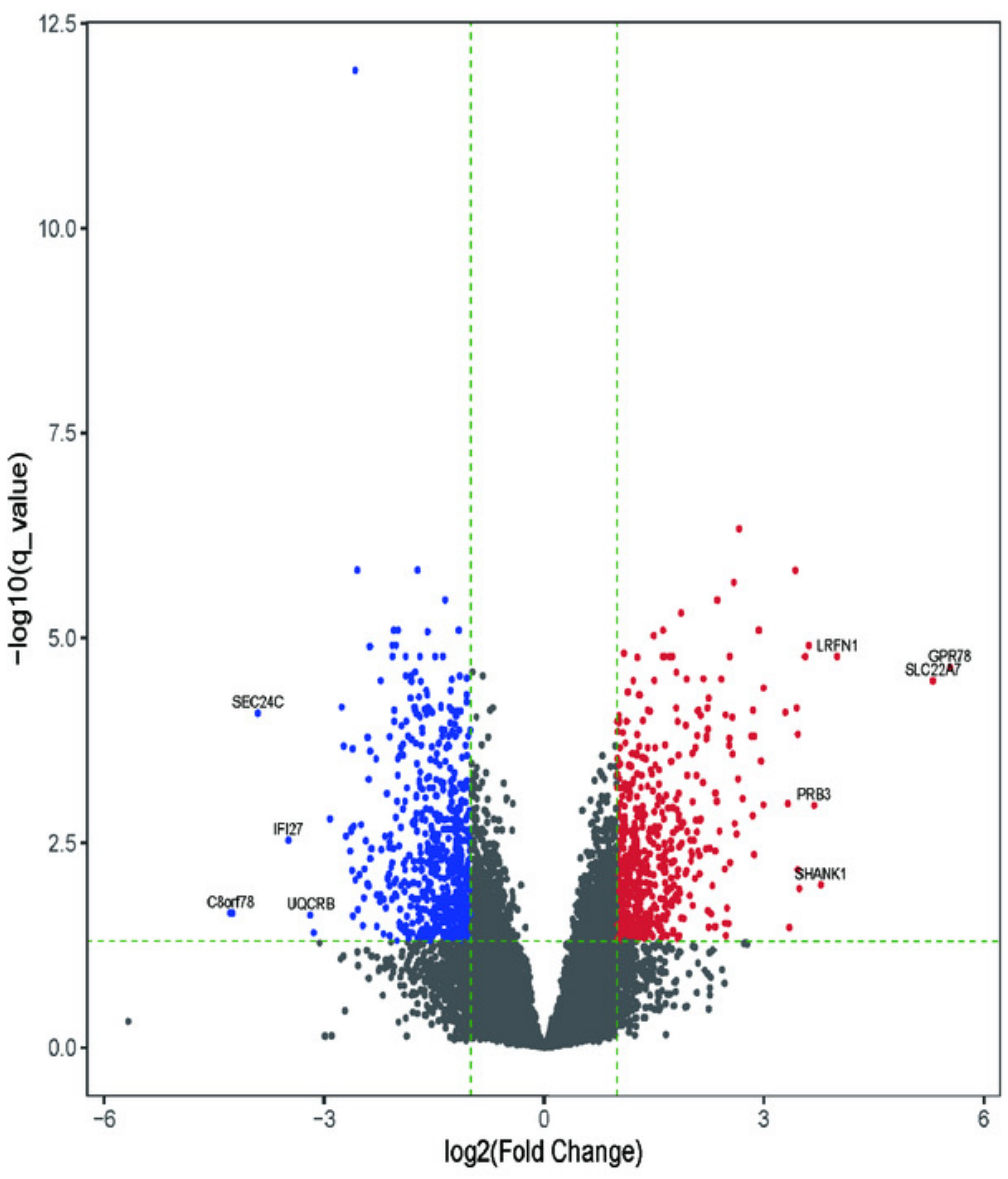

B

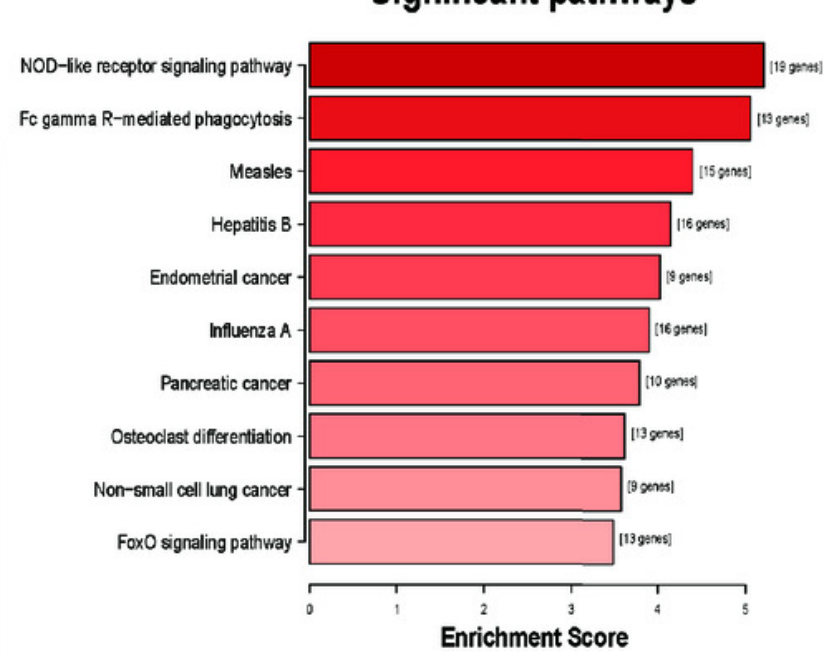

C

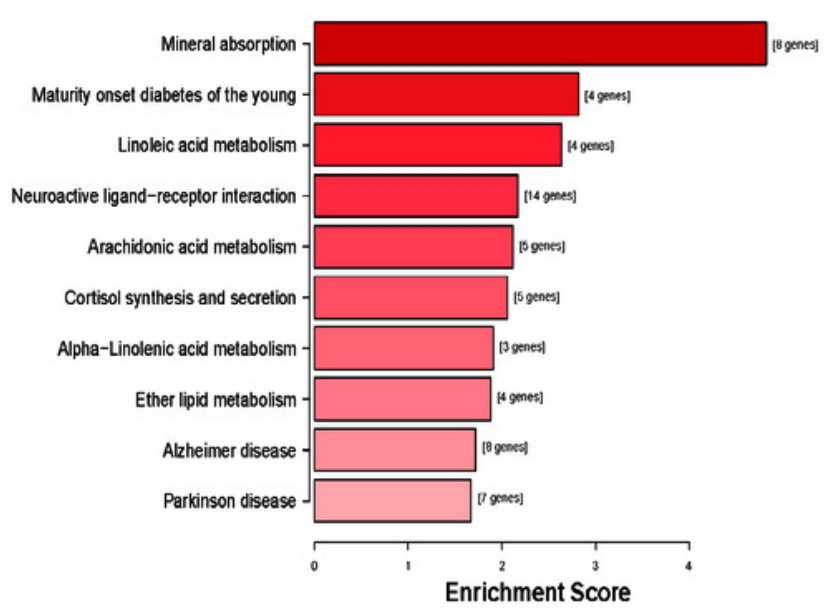




\section{Figure 4}

Construction of the ceRNA network and functional analysis of the genes in the network.

(A) Venn diagram showed the online tools were used in circRNA-miRNA corresponding relationship prediction and the (B) miRNA-mRNA corresponding prediction. (C) The circRNA-associated ceRNA network. The round nodes represent DEmRNAs, the rhombus represent DEmiRNAs, the v-type denote DEcircRNAs. The up or down-regulated genes are represented in pink and green, respectively, the darker color indicates the larger absolute value of $\log _{2}(F C)$. (D) Top 10 enrichment KEGG pathways for the related genes in the ceRNA network, the $x$-axis shows the names of the pathway, the $y$-axis shows enrichment score. (E) Top 10 GO enrichment process categorized by biological process (BP), (F) molecular function (MF), and (G) cellular component $(\mathrm{CC})$, red indicates higher enrichment, the sizes of the dots represent the numbers of genes in each GO category.
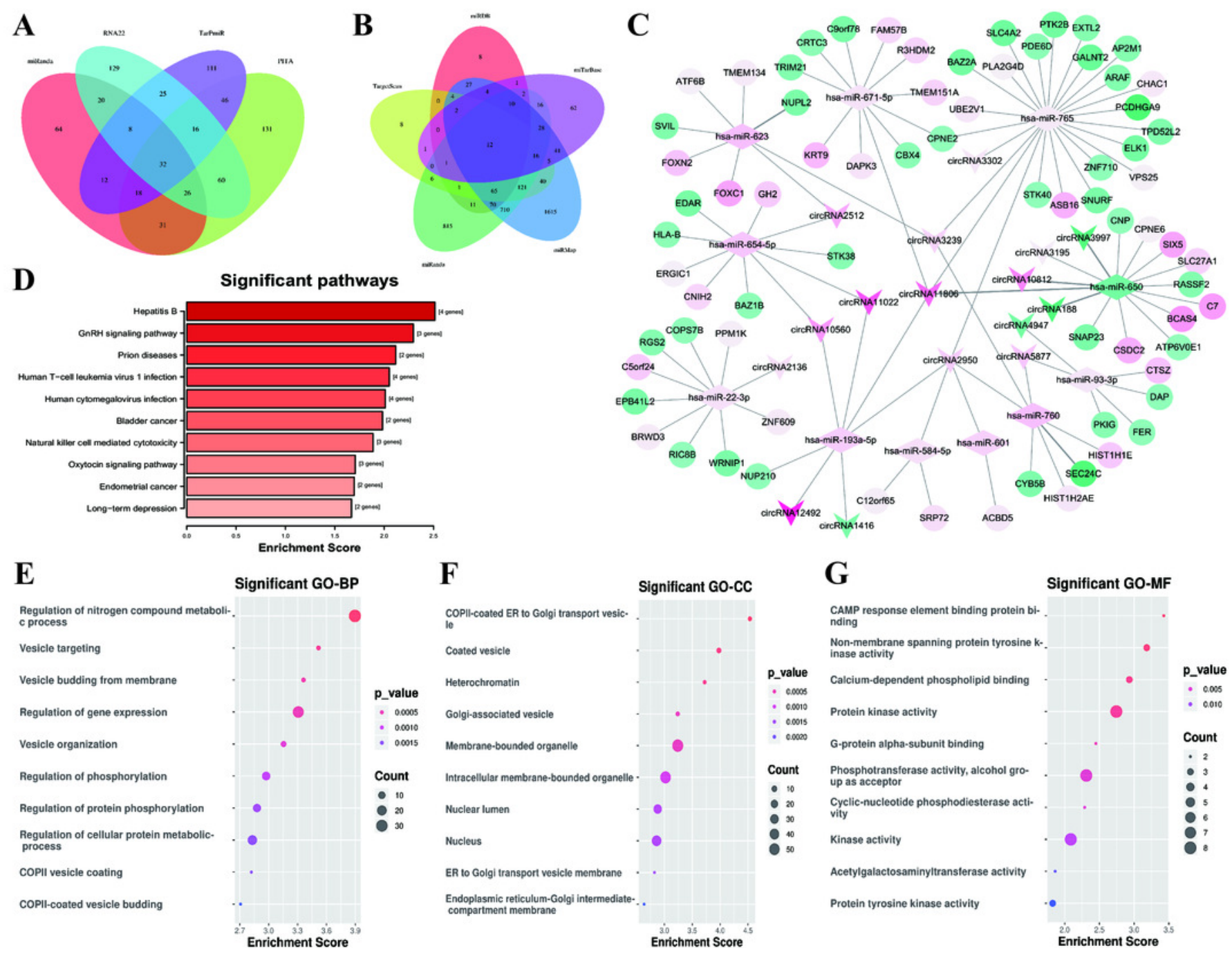


\section{Figure 5}

PPI network analysis of ceRNA-associated genes and qRT-PCR validation of the potentially hub genes.

(A) PPI network was constructed with genes from the ceRNA network, by using online tool STRING and threshold required confidence (combined score) $>0.7$. (B) The relative expression levels of ASB16, (C) HLA-B, (D) TRIM21, (E) SEC24C by qRT-PCR analysis in the PBMCs from HCs and IgAN patients ( $n=8), \beta$-actin was used as the house-keeping gene for normalization, data were expressed as a ratio of average $2-\Delta \Delta \mathrm{Ct}$, and means $\pm \mathrm{SD}$. PPI:

Protein-protein interaction. STRING: Search tool for the retrieval of interacting genes/proteins. HCs: healthy controls. 


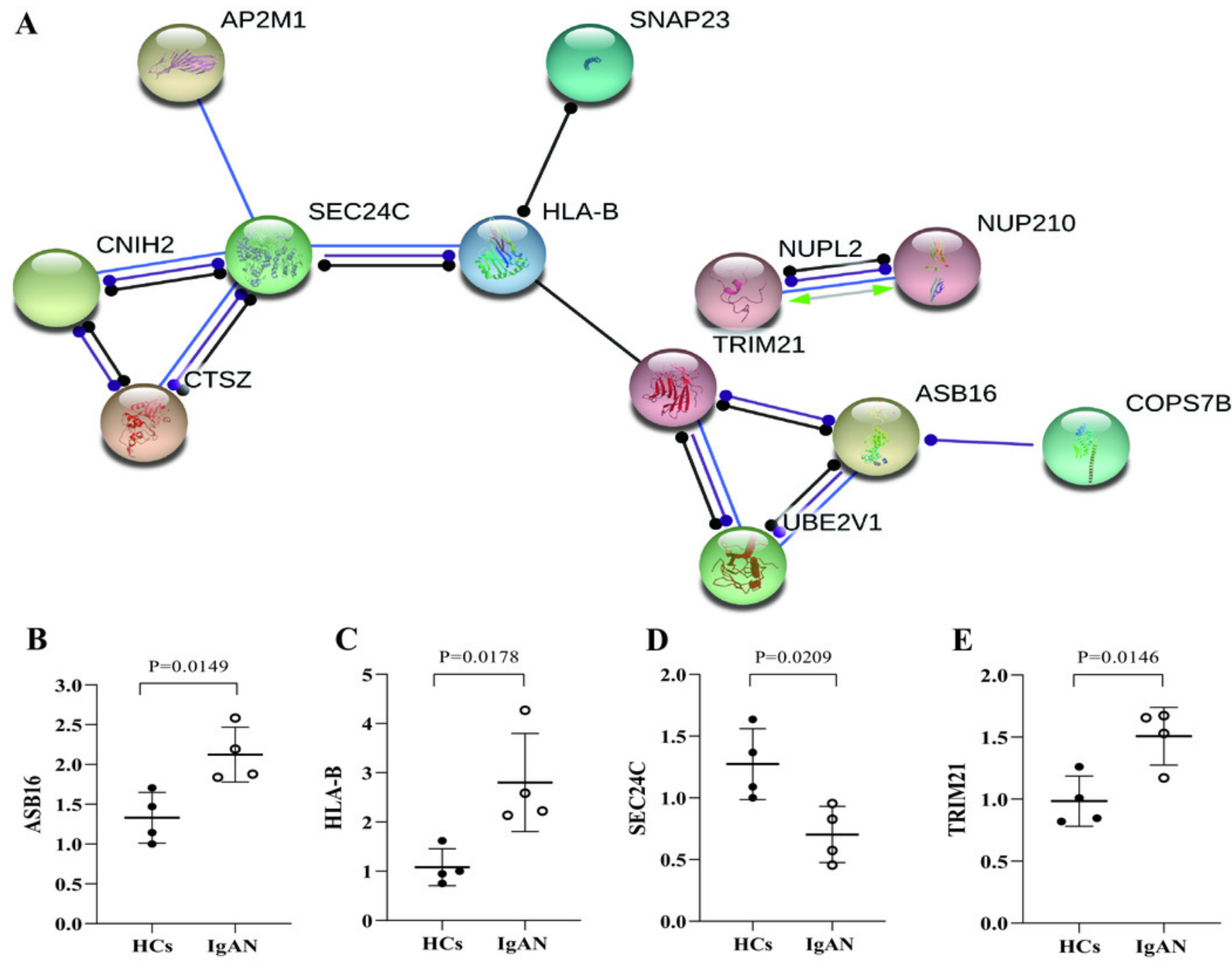




\section{Table $\mathbf{1}$ (on next page)}

10 Most dysregulated circRNA transcripts between IgAN patients and healthy controls. 
1 Table 1.10 most dysregulated circRNA transcripts between IgAN patients and healthy controls.

\begin{tabular}{|c|c|c|c|c|c|c|c|}
\hline Accession & CricBase & Gene symbo & omosome & Strand & Regulati & $\log _{2}(\mathrm{FC})$ & P-value \\
\hline circRNA9482 & hsa_circ_0038725 & IL4R & Chr16 & + & Up & 6.03134 & 0.01027 \\
\hline circRNA11137 & novel_circ & EMB & Chr5 & - & $\mathrm{Up}$ & 6.01153 & 0.00447 \\
\hline circRNA12492 & novel_circ & SP140L & Chr2 & + & Up & 5.98330 & 0.00015 \\
\hline circRNA4713 & novel_circ & TLN1 & Chr9 & - & Down & 5.69939 & 0.02184 \\
\hline circRNA10024 & novel_circ & RPS6KA5 & Chr14 & - & $\mathrm{Up}$ & 5.59830 & 0.00903 \\
\hline circRNA8550 & hsa_circ_0028670 & TAOK3 & Chr12 & - & Up & 5.46949 & 0.00979 \\
\hline circRNA10745 & hsa_circ_0007612 & ORC5 & Chr7 & - & $\mathrm{Up}$ & 5.16406 & 0.03555 \\
\hline circRNA11518 & novel_circ & HERC3 & Chr4 & + & Up & 5.13206 & 0.00143 \\
\hline circRNA4236 & hsa_circ_0004893 & PTP4A2 & Chr1 & + & Down & 4.74473 & 0.00931 \\
\hline circRNA11045 & hsa_circ_0009096 & UTRN & Chr6 & + & Up & 4.49705 & 0.03553 \\
\hline
\end{tabular}


Table 2 (on next page)

Statistical analysis of deferentially expressed circRNAs, miRNAs and mRNAs. 
1 Table 2. Statistical analysis of differentially expressed circRNAs, miRNAs and mRNAs.

\begin{tabular}{llllll}
\hline & & Differential & & Max \\
Data Resource Type of RNAs & Samples & Platforms & Expression & Threshold & $\log _{2}($ FC)
\end{tabular}

\begin{tabular}{cccccccccccc}
\hline & & IgAN & Control & & Up & Down & log2(FC) P-value & Up & Down \\
\hline RNA-seq & circRNAs & 3 & 3 & GPL20301 & 112 & 33 & 1 & 0.05 & 6.03 & -6.01 \\
GSE25590 & miRNAs & 7 & 7 & GPL7731 & 18 & 4 & 1 & 0.05 & 2.74 & -1.99 \\
GSE73953 & mRNAs & 15 & 2 & GPL4133 & 522 & 595 & 1 & 0.05 & 5.54 & -4.27 \\
\hline
\end{tabular}

2

3

4 